\title{
In vitro inhibition of Plasmodium falciparum early and late stage gametocyte viability by extracts from eight traditionally used South
}

\section{African plant species}

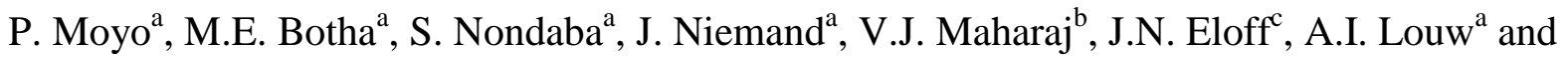
L. Birkholtz ${ }^{\mathrm{a}, *}$

${ }^{a}$ Department of Biochemistry and ${ }^{b}$ Department of Chemistry, Faculty of Natural and Agricultural Sciences, Centre for Sustainable Malaria Control, University of Pretoria, Private Bag x20, Pretoria 0028, South Africa

${ }^{c}$ Phytomedicine Programme, Department of Paraclinical Sciences, Faculty of Veterinary Science, University of Pretoria, Private Bag x04, Onderstepoort 0110, Pretoria, South Africa

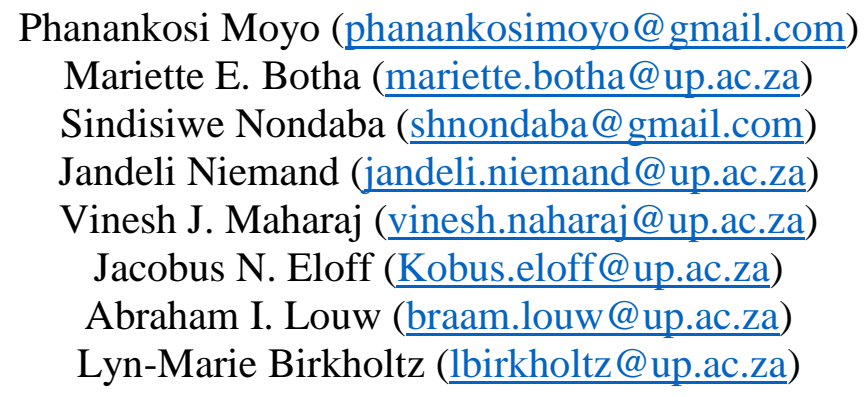

*Corresponding author: L. Birkholtz

Tel:+27 12420 2479;

Fax: +27 123625302 .

E-mail address: lbirkholtz@up.ac.za

Postal address:

Department of Biochemistry

Faculty of Natural and Agricultural Sciences

University of Pretoria,

Private bag, x20

Hatfield 0028

Pretoria, Gauteng

SOUTH AFRICA 


\begin{abstract}
Ethnopharmacological relevance: Extracts of plant species, used traditionally to treat malaria, have been extensively investigated for their activity against Plasmodium intraerythrocytic asexual parasites in search of new antimalarial drugs. However, less effort has been directed towards examining their efficacy in blocking transmission. Here, we report the results of the in vitro screening of extracts from eight selected plant species used traditionally to treat malaria in South Africa for activity against $P$. falciparum NF54 early and late stage gametocytes. The species used were Khaya anthotheca, Trichilia emetica, Turraea floribunda, Leonotis leonurus, Leonotis leonurus ex Hort, Olea europaea subsp. Africana, Catha edulis and Artemisia afra.
\end{abstract}

Aim of the Study: To investigate the activities of extracts from plant species traditionally used for malaria treatment against $P$. falciparum gametocytes.

Material and Methods: Air-dried and ground plant leaves were extracted using acetone. Primary two point in vitro phenotypic screens against both early and late stage gametocytes were done at 10 and $20 \mu \mathrm{g} / \mathrm{ml}$ followed by full $\mathrm{IC}_{50}$ determination of the most active extracts. Inhibition of gametocyte viability in vitro was assessed using the parasite lactate dehydrogenase (pLDH) assay.

Results: Of the eight crude acetone extracts from plant species screened in vitro, four had good activity with over 50-70\% inhibition of early and late stage gametocytes' viability at 10 and $20 \mu \mathrm{g} / \mathrm{ml}$, respectively. Artemisia afra (Asteraceae), Trichilia emetica (Meliaceae) and Turraea floribunda (Meliaceae) were additionally highly active against both gametocyte stages with $\mathrm{IC}_{50}$ values of less than $10 \mu \mathrm{g} / \mathrm{ml}$ while Leonotis leonurus ex Hort (Lamiaceae) was moderately active $\left(\mathrm{IC}_{50}<20 \mu \mathrm{g} / \mathrm{ml}\right.$ ). The activity of these three highly active plant species was significantly more pronounced on late stage gametocytes compared to early stages.

Conclusion: This study shows the potential transmission blocking activity of extracts from selected South African medicinal plants and substantiates their traditional use in malaria control that broadly encompasses prevention, treatment and transmission blocking. Further studies are needed to isolate and identify the active principles from the crude extracts of $A$. afra, T. emetica and $T$. floribunda, as well as to examine their efficacy towards blocking parasite transmission to mosquitoes. 


\section{Graphical Abstract}

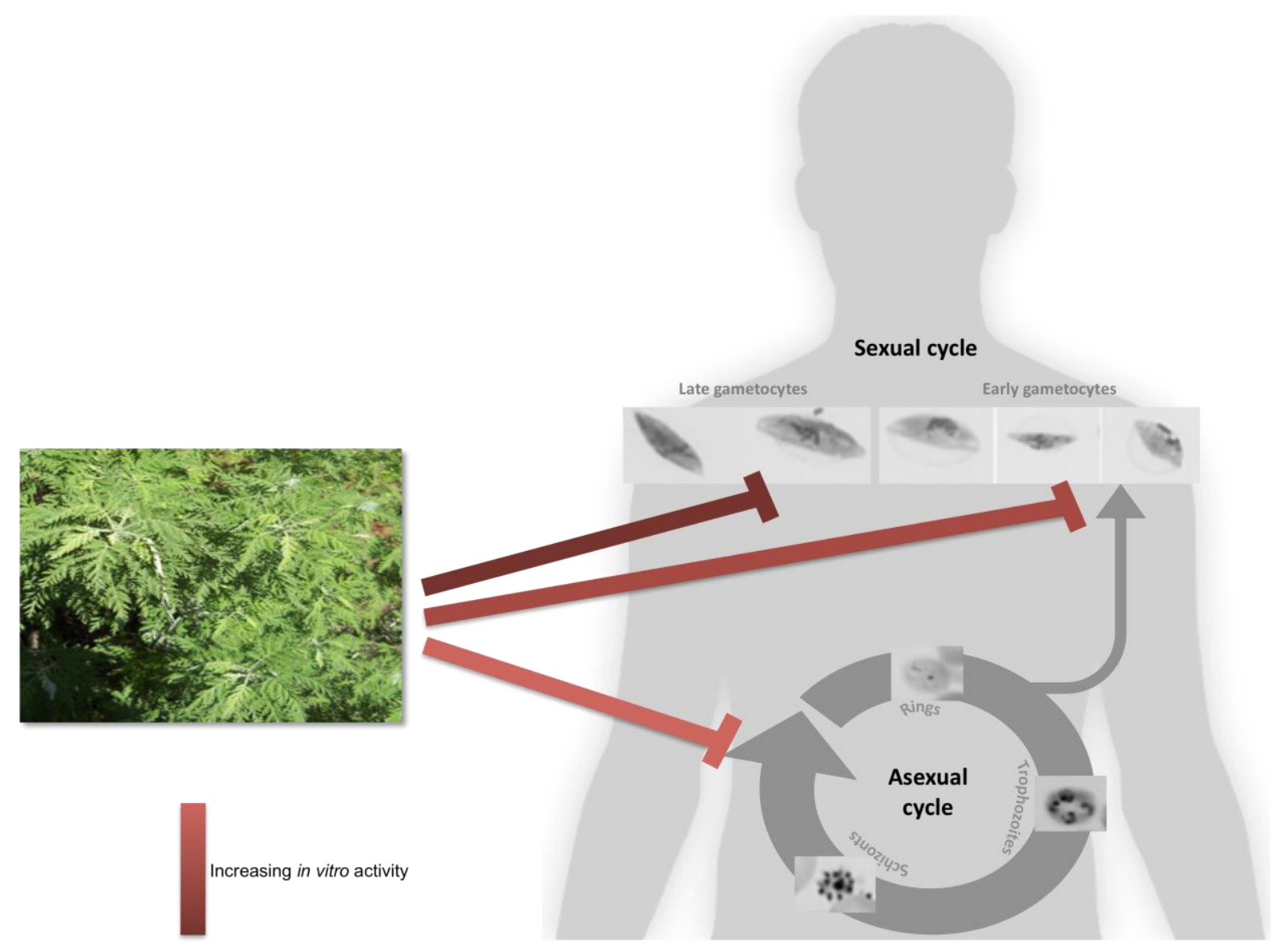

Keywords: Gametocytes; Plant Leaf Extracts; Malaria; Plasmodium falciparum 
For the past decades, the management of malaria has primarily relied on vector control and chemotherapeutic drugs such as chloroquine and the artemisinin-based combination therapies (ACT's). While the benefits of adopting these measures have been evident (Bhatt et $a l ., 2015)$, unfortunately so too has been the emergence of insecticide resistant mosquitoes (Benelli, 2015; Dai et al., 2015; Edi et al., 2012; Ranson et al., 2011) and drug resistant strains of Plasmodium falciparum parasites (Ashley et al., 2014; Dondorp et al., 2009; Tun et $a l ., 2015)$. There is thus a dire need to advance the efficacy of these approaches with novel interventions, drugs and insecticides in the fight against this disease. In the current era of renewed calls for malaria elimination and eradication, there is a need to devise new strategies to complement current interventions (Roberts and Enserink, 2007). One such identified strategy is to block transmission from the human host to the mosquito vector (Alonso et al., 2011).

Human-to-mosquito transmission blocking entails targeting the sexual stages of the parasite, the gametocytes. This is the only stage of Plasmodium that can infect a female Anopheles mosquito for the sexual development of the parasite into sporozoites that can in turn infect humans and thereby perpetuate the lifecycle (Baker, 2010). Inhibiting gametocyte development would significantly reduce the number of infective mosquitoes and thus the number of newly infected patients. A single low dose primaquine $(0.25 \mathrm{mg} / \mathrm{kg})$ remains the only gametocytocidal drug recommended by the World Health Organisation against $P$. falciparum gametocytes (White et al., 2014). However, its use is restricted due to adverse effects on patients with glucose-6-phosphate dehydrogenase deficiency (Baird and Hoffman, 2004), a genetic disorder that is prevalent among populations in malaria endemic areas (Nkhoma et al., 2009). While some antimalarial drugs are active against the early stages of $P$. falciparum gametocytes (Abay, 2013; Butcher, 1997; Lucantoni et al., 2013; Mackerras and Ercole, 1949; Price et al., 1996; White et al., 2014), they are not effective at clinically relevant concentrations on the late stage, transmittable gametocytes. There is therefore an urgent need to find new safe and efficacious transmission blocking drugs that can target late stage gametocytes. 
Plant-derived natural products have played a fundamental role in the control of malaria. They have been a vital source of some of the mainstay drugs in malaria treatment such as the alkaloid, quinine and sesquiterpene lactone, artemisinin (Wells, 2011). These drugs were identified following intensive screens of hundreds of plant extracts for activity against the intraerythrocytic, asexual parasites of Plasmodium (Tu, 2011; Zhang, 2011). By contrast, less effort has been channeled towards examining extracts of plants for their activity against the sexual stages of the parasite. Currently, only three plant species have been directly screened for their malaria transmission blocking capacity: Azadirachta indica (Meliaceae) (Dhar et al., 1998; Jones et al., 1994; Lucantoni et al., 2010; Udeinya et al., 2006, 2008; Yerbanga et al., 2014), Vernonia amygdalina (Asteraceae) (Abay et al., 2015, 2013) and Guiera senegalensis (Combretaceae) (Yerbanga et al., 2014). A. indica has been comprehensively studied and shown to have good gametocytocidal activity in vitro (Dhar et al., 1998; Jones et al., 1994; Udeinya et al., 2008, 2006), in vivo (Lucantoni et al., 2010) and ex vivo (Yerbanga et al., 2014). The active components are a diverse range of limonoid compounds (Jones et al., 1994; Lucantoni et al., 2010; Yerbanga et al., 2014). V. amygdalina gametocytocidal activity was demonstrated in vivo with the active principles identified as the sesquiterpene lactones vernadalol and vernolide (Abay et al., 2015, 2013). G. senegalensis did not have any transmission blocking properties (Yerbanga et al., 2014).

Even given the complexities involved in plant-based screening for antimalarial activity, the paucity of information of plant species with potency against early and late stage gametocytes encourages studies to identify malaria transmission blocking capabilities of plant extracts. In the current study, we explored the anti-gametocyte properties of eight plant species traditionally used to treat malaria in South Africa. This was investigated by using a stage-specific in vitro phenotypic screen of crude extracts of plants for activity against early and late stage $P$. falciparum NF54 gametocytes. 


\subsection{Plant selection, collection and crude acetone extract preparation}

The subset of eight plant species investigated in this study was selected as follows: Plants were first and foremost chosen for being used traditionally for either malaria or fever treatment as documented in ethnobotanical studies (Alam et al., 2012; Clarkson et al., 2004; Van Wyk, 2008; Table 1). From the ethnobotanical set, plants were selected based on two parallel criteria: 1) previous reports of either good or moderate activity against the intraerythrocytic asexual parasites of $P$. falciparum parasites $\left(\mathrm{IC}_{50} \leq 10 \mu \mathrm{g} / \mathrm{ml}-\right.$ good; $20 \geq \mathrm{IC}_{50}>10$ - moderate) and 2) evidence of the presence of compounds (at plant family level) that are structurally similar to those known to be gametocytocidal either in vitro, in vivo or ex vivo (Abay et al., 2015; Adjalley et al., 2011; D’Alessandro et al., 2013; Duffy and Avery, 2013; Jones et al., 1994; Lucantoni et al., 2013, 2010; Saenz et al., 2013; Sun et al., 2013; Yerbanga et al., 2014). Using these two parallel selection strategies, the ethnobotanical set was narrowed down to a hundred plant species from which eight were collected and screened in the current study.

Leaves of the eight plant species were collected from the Manie van der Schijff Botanical Garden at the University of Pretoria, Hatfield campus in July 2014. Plants were identified by a curator and voucher specimens were made and deposited at the H.G.W.J. Schweickerdt Herbarium of the University of Pretoria (Table 1) and confirmed on www.theplantlist.org. Collected plant material was air dried, ground to fine powder and extracted in acetone as described elsewhere (Dzoyem and Eloff, 2015). Phytochemical profiling of dry crude extracts was performed using thin layer chromatography (TLC) (Kotze and Eloff, 2002).

\subsection{In vitro cultivation of asexual and sexual stage P. falciparum parasites}

The chloroquine sensitive and high gametocyte yielding $P$. falciparum NF54 strain was established in human erythrocytes $\left(\mathrm{O}^{+}\right.$, suspended at $5 \%$ haematocrit $)$, cultured asexually and synchronised at least two times on ring stage parasites.

Gametocytogenesis and gametocyte culturing was performed as described by Reader et al. (2015). Contaminating asexual cultures were removed by $\mathrm{N}$-acetyl glucosamine (NAG) (50 mM) treatment for 96 hours (Reader et al., 2015). Gametocyte development from stages I to $\mathrm{V}$ was monitored daily by microscopic examination of Giemsa-stained smears of cultures. A suspension of uninfected erythrocytes was co-cultured along with infected cell cultures 
under similar conditions and used as background attributable to uninfected erythrocytes for the gametocyte assays.

\subsection{In vitro assessment of gametocyte viability}

The ability of extracts from plants to inhibit $P$. falciparum early and late stage gametocyte viability was assessed in vitro using the parasite lactate dehydrogenase ( $\mathrm{pLDH}$ ) assay. Modified protocols of the method of Makler and Hinrichs (1993) were used to set up the assays (D'Alessandro et al., 2015, 2013) and for examining gametocyte viability spectrophotometrically (Reader et al., 2015).

Dried crude acetone extracts were dissolved in DMSO as stock solutions and diluted in culture medium prior to their use in assays (highest concentration of DMSO to which parasites were exposed to was $0.4 \%(\mathrm{v} / \mathrm{v})$ which is non-toxic to gametocytes (D'Alessandro et al., 2015, 2013; Duffy and Avery, 2013; Lucantoni et al., 2013). These extracts were transferred into 96 well sterile plates and seeded with an equal volume (100 $\mu 1 /$ well) of gametocyte suspension (0.8-3\% gametocytaemia) to achieve a final haematocrit of $1 \%$ (D’Alessandro et al., 2013). Dual primary point screens (using 10 and $20 \mu \mathrm{g} / \mathrm{ml}$ plant extract) were followed by the determination of the $\mathrm{IC}_{50}$ values (concentration of extract required to inhibit gametocyte viability by $50 \%$ ) using a range of starting concentrations of the most active plant extracts (final concentration range of $0.156-40 \mu \mathrm{g} / \mathrm{ml}$ ). Methylene blue $(10 \mu \mathrm{M}$ ) served as the positive drug control.

Plates were incubated at $37^{\circ} \mathrm{C}$ for 72 hours, followed by the replacement of spent medium with extract-free culture medium (75\% medium change) (D'Alessandro et al., 2015, 2013). Plates were then incubated for a further $72 \mathrm{hrs}$ before assessing viability by measuring pLDH activity (Reader et al., 2015). All assays were done in technical triplicates with three independent biological repeats each. Non-linear regression analysis (using GraphPad Prism, version 5.0) was used to determine $\mathrm{IC}_{50}$ values.

\subsection{In vitro antiplasmodial activity against intraerythrocytic asexual parasites}

The activity was determined as described by Clarkson et al. (2003). Dual point screens were set up at 10 and $20 \mu \mathrm{g} / \mathrm{ml}$. Chloroquine $(0.5 \mu \mathrm{M})$ was used as positive drug control. 


\section{Results}

\subsection{Plant selection, collection and extraction}

Eight plant species representing five different families and seven genera were collected for the study. The three plant species investigated namely, L. leonurus, L. leonurus ex Hort and Olea europaea (Oleaceae) were selected based on previously reported activity against the intraerythrocytic asexual parasites of P. falciparum (Table 1) (Clarkson et al., 2004). Khaya anthotheca (Meliaceae) was selected as the Meliaceae family, has been shown to produce limonoids that are potent against gametocytes (Jones et al., 1994; Lucantoni et al., 2010; Yerbanga et al., 2014). The remainder of plant species was selected based on both activity and natural compound production (Table 1). Different extraction yields (grams acetone plant extract/grams dry plant starting material) were obtained, with $K$. anthotheca producing the highest yield (11\%) while T. emetica had the lowest ( 3\%; Table 1). Major compound classes identified through TLC phytochemical profiling included phenols, terpenoids and stilbenes. A. afra was the only species observed to have compounds that fluoresce under UV light (Fig. $1 \mathrm{~S})$.

Table 1. Plant species selected and evaluated for potential inhibition of asexual or sexual stage $P$. falciparum parasites.

\begin{tabular}{|c|c|c|c|c|}
\hline Plant specie (Family) & Common names & $\begin{array}{c}\text { Selection } \\
\text { criteria }\end{array}$ & $\begin{array}{l}\text { Extraction } \\
\text { Yield }(\%)\end{array}$ & $\begin{array}{l}\text { Voucher } \\
\text { No. }\end{array}$ \\
\hline $\begin{array}{l}\text { Khaya anthotheca (Welw) } \\
\text { C.DC. (Meliaceae) }\end{array}$ & $\begin{array}{l}\text { Rooimahonie (Afrikaans), red } \\
\text { mahogany (English) }\end{array}$ & $\mathrm{c}$ & 11.05 & PRU 121391 \\
\hline $\begin{array}{l}\text { Trichilia emetica Vahl } \\
\text { subsp. Emetica } \\
\text { (Meliaceae) }\end{array}$ & $\begin{array}{l}\text { Umkhulu (Zulu), bosveldrooiessenhout } \\
\text { (Afrikaans) }\end{array}$ & $\mathrm{a}, \mathrm{c}$ & 3.25 & PRU 121390 \\
\hline $\begin{array}{l}\text { Turraea floribunda Hochst. } \\
\text { (Meliaceae) }\end{array}$ & $\begin{array}{l}\text { Umadlozane (Zulu), umhlatholana } \\
\text { (Xhosa), kanferfoelieboom (Afrikaans) }\end{array}$ & $\mathrm{a}, \mathrm{c}$ & 7.12 & PRU 121387 \\
\hline $\begin{array}{l}\text { Leonotis leonurus (L.) R. Br. } \\
\text { (Lamiaceae) }\end{array}$ & $\begin{array}{l}\text { Wilde dagga (Afrikaans), lion’s ear } \\
\text { (English) }\end{array}$ & $\mathrm{a}$ & 5.85 & PRU 121393 \\
\hline $\begin{array}{l}\text { Leonotis leonurus ex Hort } \\
\text { (yellow) (Lamiaceae) }\end{array}$ & Wilde dagga (Afrikaans) & a & 3.85 & PRU 121394 \\
\hline $\begin{array}{l}\text { Olea europaea subsp. } \\
\text { Africana (Oleaceae) }\end{array}$ & $\begin{array}{l}\text { Wild olive (English), Olienhout } \\
\text { (Afrikans) }\end{array}$ & $\mathrm{b}$ & 7.08 & PRU 121388 \\
\hline $\begin{array}{l}\text { Catha edulis (Vahl) Endl } \\
\text { (Celastraceae) }\end{array}$ & $\begin{array}{l}\text { Umhlwazi (Zulu), igqwaka (Xhosa), } \\
\text { khat (Afrikaans) }\end{array}$ & $\mathrm{a}$ & 3.33 & PRU 121392 \\
\hline $\begin{array}{l}\text { Artemisia afra Jacq. ex } \\
\text { Wild. (Asteraceae) }\end{array}$ & $\begin{array}{l}\text { African wormwood (English), Wilde- } \\
\text { als (Afrikaans), Umhlonyane (Zulu) }\end{array}$ & $\mathrm{a}, \mathrm{c}$ & 6.38 & PRU 121389 \\
\hline
\end{tabular}




\subsubsection{Gametocyte viability (Dual point screens)}

The effects of crude acetone extracts of eight different plants on early and late stage gametocyte viability were assessed in vitro using a pLDH assay (Fig. 1). Gametocyte cultures used for both early and late stage assays contained $>85 \%$ of each respective stage being investigated (Fig. 1A), validating their use in the stage-specific evaluation of gametocytocidal activity analysis. Furthermore, quality evaluation of assay performance revealed that for both stage-specific assays, a signal-to-background ( $\mathrm{S} / \mathrm{B})$ ratio of $>3$ was obtained; as well as acceptable signal-to-noise ratios $(\mathrm{S} / \mathrm{N}>20)$ (Fig. 1B). Assay reproducibility was high as indicated by $\mathrm{Z}$ '-factors being consistently $>0.8$.

A

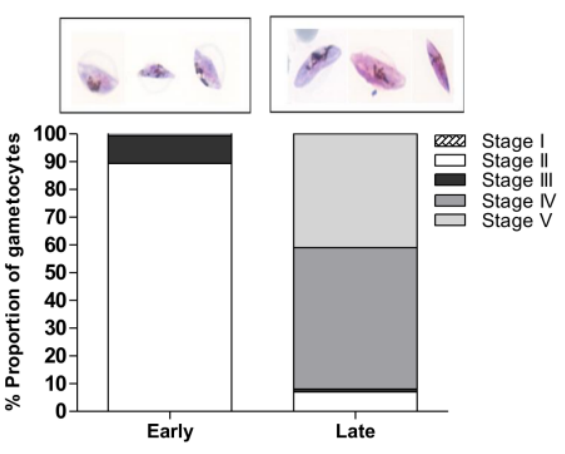

C

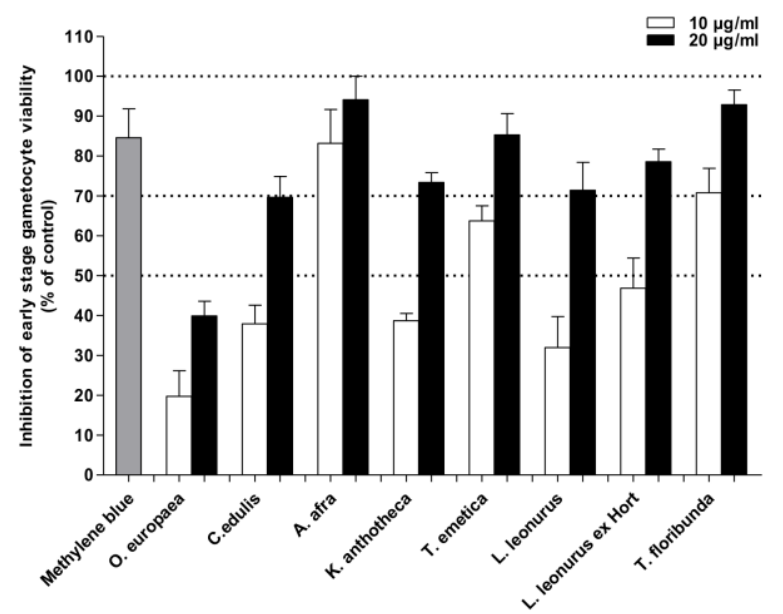

B

\begin{tabular}{lcc}
\hline $\begin{array}{c}\text { Gametocyte } \\
\text { stages }\end{array}$ & Assay performance quality indicators \\
\hline & $\mathrm{S} / \mathrm{B}$ & $\mathrm{S} / \mathrm{N}$ \\
\cline { 2 - 3 } Early & $3.1 \pm 0.18$ & $24.3 \pm 10.82$ \\
Late & $3.7 \pm 0.73$ & $220.8 \pm 71.72$ \\
\hline
\end{tabular}

D

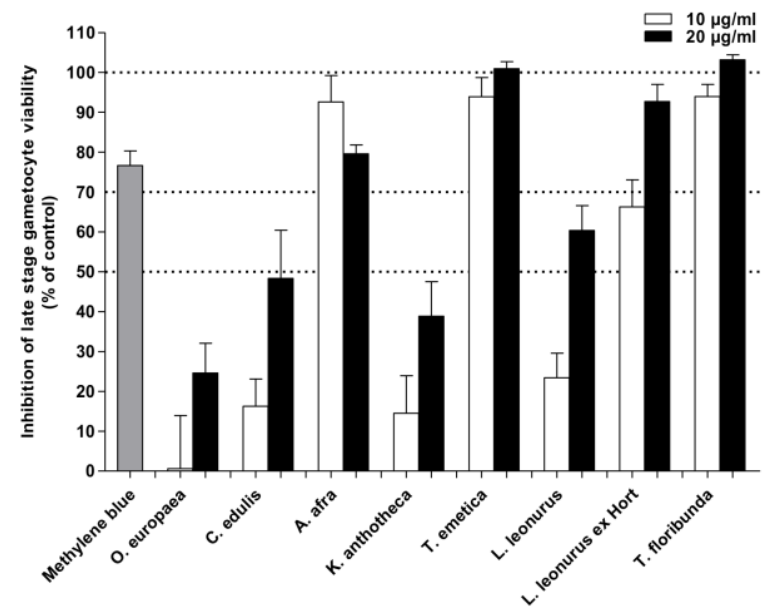

Figure 1. In vitro inhibition of early and late stage $P$. falciparum NF54 gametocyte viability by crude acetone extract of plants. (A) Stage-specific distribution of gametocyte populations in cultures used for dual point screens. (B) Assay quality indicator parameters. Dual point primary screens of eight different plant extracts at 10 and $20 \mu \mathrm{g} / \mathrm{ml}$ on (C) early and (D) late stage gametocytes (0.8-3\% gametocytaemia), evaluated with a $72+72 \mathrm{hr}$ pLDH assay. Data have been normalised against untreated gametocyte controls. Positive control drug used was methylene blue $(10 \mu \mathrm{M})$. Results are the mean \pm SEM of three independent biological repeats each done in triplicate. 
Dual point evaluation (10 and $20 \mu \mathrm{g} / \mathrm{ml}$ ) of crude extracts of the eight plant species on the in vitro viability of early stage gametocytes indicated dose dependent inhibition by the crude plant extracts (Fig. 1C). This reduction ranged between $20 \%$ to $94 \%$ with O. europaea and $A$. afra extracts having the lowest and highest activities, respectively. While seven plant extracts produced $>70 \%$ inhibition of early stage gametocyte in vitro viability at $20 \mu \mathrm{g} / \mathrm{ml}$, only three plant extracts (A. afra, T. emetica and T. floribunda) showed $>50 \%$ inhibition at $10 \mu \mathrm{g} / \mathrm{ml}$ (Fig. 1C).

Results for late stage gametocyte screens mirrored those of early stages with a few exceptions. The plant extracts showed concentration dependent pattern of inhibition ranging from $99.4 \%$ to $0 \%$ (Fig. 1D). Extract from O. europaea was the least active while A. afra, T. emetica, T. floribunda and L. leonurus ex Hort extracts were the most potent. K. anthotheca, O. europaea and C. edulis extracts had a lower activity against the late stage gametocytes in comparison to the early stages. By contrast, A. afra, T. emetica, T. floribunda and L. leonurus ex Hort extracts demonstrated increased activity. The range of inhibition of gametocyte viability in vitro for these four species of plants was from $47-94 \%$ to $66-100 \%$ against the early and late stage gametocytes, respectively (Fig. 1C and D). Only four plant extracts $(A$. afra, T emetica, T. floribunda and L. leonurus ex Hort) displayed over $50 \%$ and $70 \%$ reduction on viability at either 10 or $20 \mu \mathrm{g} / \mathrm{ml}$, respectively, against the late stage gametocytes (Fig. 1D). From the dual point assays, A. afra, T. emetica, T. floribunda and $L$. leonurus ex Hort extracts were selected for full dose-response and $\mathrm{IC}_{50}$ determination.

\subsubsection{Gametocyte viability ( $I C_{50}$ determination)}

Three of the four plant species investigated in the study exhibited $\mathrm{IC}_{50}$ values of less than $10 \mu \mathrm{g} / \mathrm{ml}$ against both early and late stage gametocytes of $P$. falciparum (Fig. 2). Methylene blue as positive drug control had in vitro gametocyte viability inhibition values of $88.2 \pm 1.1 \%$ and $87.4 \pm 2.7 \%$ against early and late stages, respectively. The assay performance for doseresponse evaluation remained consistent with $\mathrm{S} / \mathrm{B}$ and $\mathrm{S} / \mathrm{N}$ ratios of 3-4 and 53-111, respectively.

Of the four selected plants species, A. afra was the most potent with $\mathrm{IC}_{50}$ values of $5.7 \pm 0.3$ and $3.2 \pm 0.2 \mu \mathrm{g} / \mathrm{ml}$ against early and late stage gametocytes, respectively. This was closely followed by $T$. emetica $(7.6 \pm 0.9 \mu \mathrm{g} / \mathrm{ml}$ - early stages; $3.8 \pm 0.5 \mu \mathrm{g} / \mathrm{ml}$ - late stages) and T. floribunda $(9.2 \pm 0.2 \mu \mathrm{g} / \mathrm{ml}$ - early stages; $4.6 \pm 0.7 \mu \mathrm{g} / \mathrm{ml}$ - late stages $)$ while $L$. leonurus ex Hort showed moderate activity $(12.8 \pm 1.4 \mu \mathrm{g} / \mathrm{ml}$ - early stages; $13.9 \pm 2.6 \mu \mathrm{g} / \mathrm{ml}-$ 
late stages). Interestingly, three of the four plant extracts tested (A. afra, T. emetica and $T$. floribunda) exhibited significantly higher $(n=3, P<0.05$, unpaired Student's $t$-test $)$ inhibition of late stage gametocyte viability in comparison to early stage gametocyte viability, as indicated by an approximately two-fold lower $\mathrm{IC}_{50}$ value (Fig. 2).

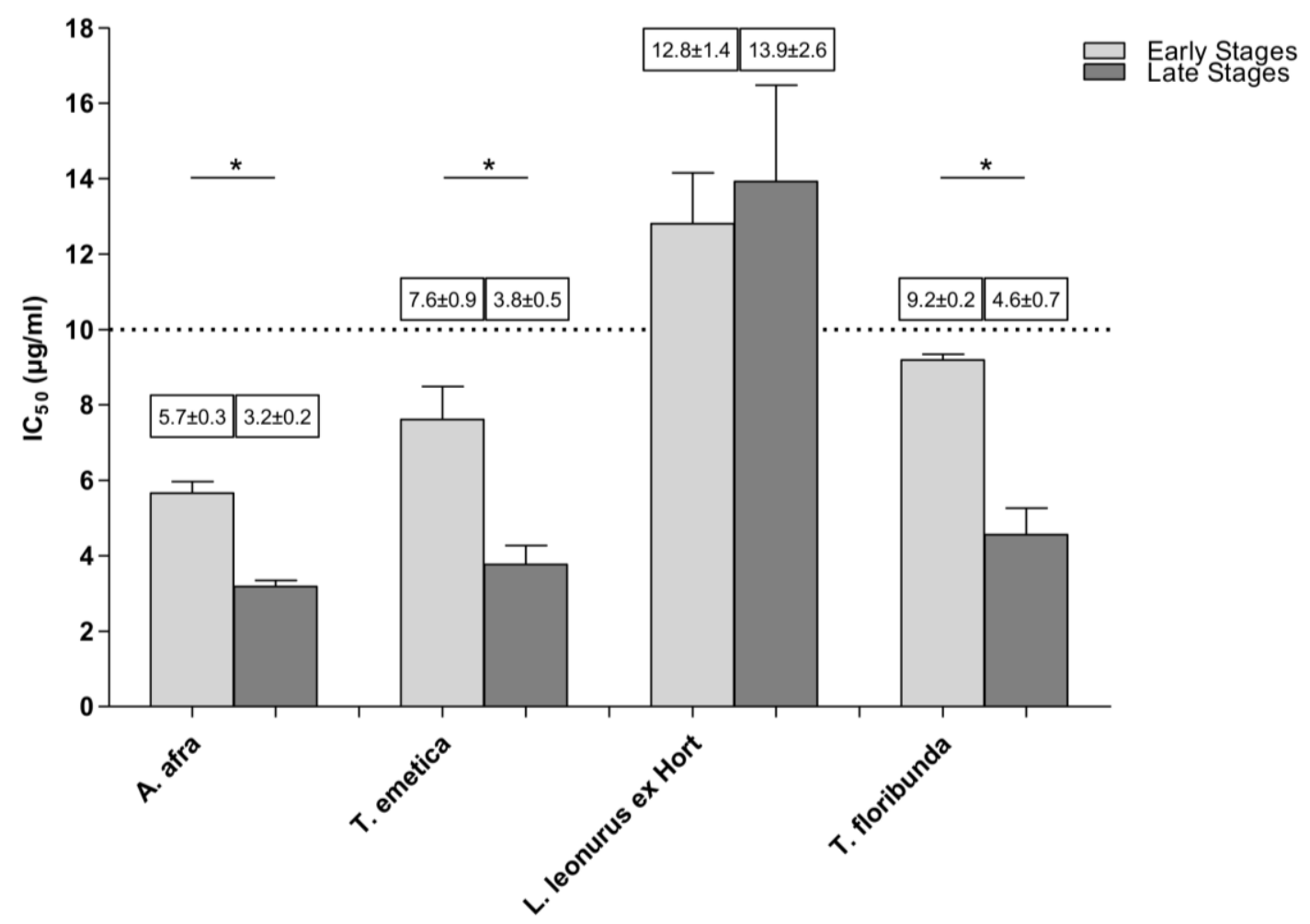

Figure 2. Dose-response evaluation of selected plant extracts against early and late stage $\boldsymbol{P}$. falciparum NF54 gametocytes. $\mathrm{IC}_{50}$ values (in $\mu \mathrm{g} / \mathrm{ml}$ ) for plant extracts on early and late stage gametocytes $(0.8-3 \%$ gametocytaemia), determined using the $72+72 \mathrm{hr}$ pLDH assay. Positive drug control was methylene blue. Data are the mean \pm SEM of three independent biological repeats each done in triplicate. Statistically significant differences between the $\mathrm{IC}_{50}$ values obtained against early compared to late stage gametocytes are indicated $(* P$ $<0.05)$ as determined by Student's $t$-test.

\subsubsection{Activity of plant extracts on gametocytes in comparison to asexual parasites}

Plant extracts were additionally evaluated for their ability to block the proliferation of intraerythrocytic asexual $P$. falciparum NF54 parasites, compared to their inhibition of viability of both early and late stage gametocytes (Fig. 3). With varying degrees of potency, the plant extracts displayed polypharmacology by inhibiting both asexual parasites and early stage gametocytes (Fig. 3A). O. europaea extracts was poorly active against both gametocyte stages as well as asexual parasites (Fig. 3A). By contrast, T. floribunda, A. afra, T. emetica, L. leonurus ex Hort, L. leonurus and C. edulis extracts were all highly active against both asexual parasites and early stage gametocytes ( $\geq 70 \%$ inhibition of viability at $20 \mu \mathrm{g} / \mathrm{ml})$. $K$. 
anthotheca extracts was highly active on the early stage gametocytes while being moderately effective against asexual parasites (between $50-70 \%$ inhibition of viability at $20 \mu \mathrm{g} / \mathrm{ml}$ ).

Comparison of the inhibitory activity of plant extracts between late stage gametocytes and asexual parasites showed similarities to that of early gametocyte stages. A. afra, T. emetica, T. floribunda and L. leonurus ex Hort extracts were the most active against both late stage gametocytes as well as asexual parasites, exhibiting over $80 \%$ inhibition in both instances (Fig. 3B).

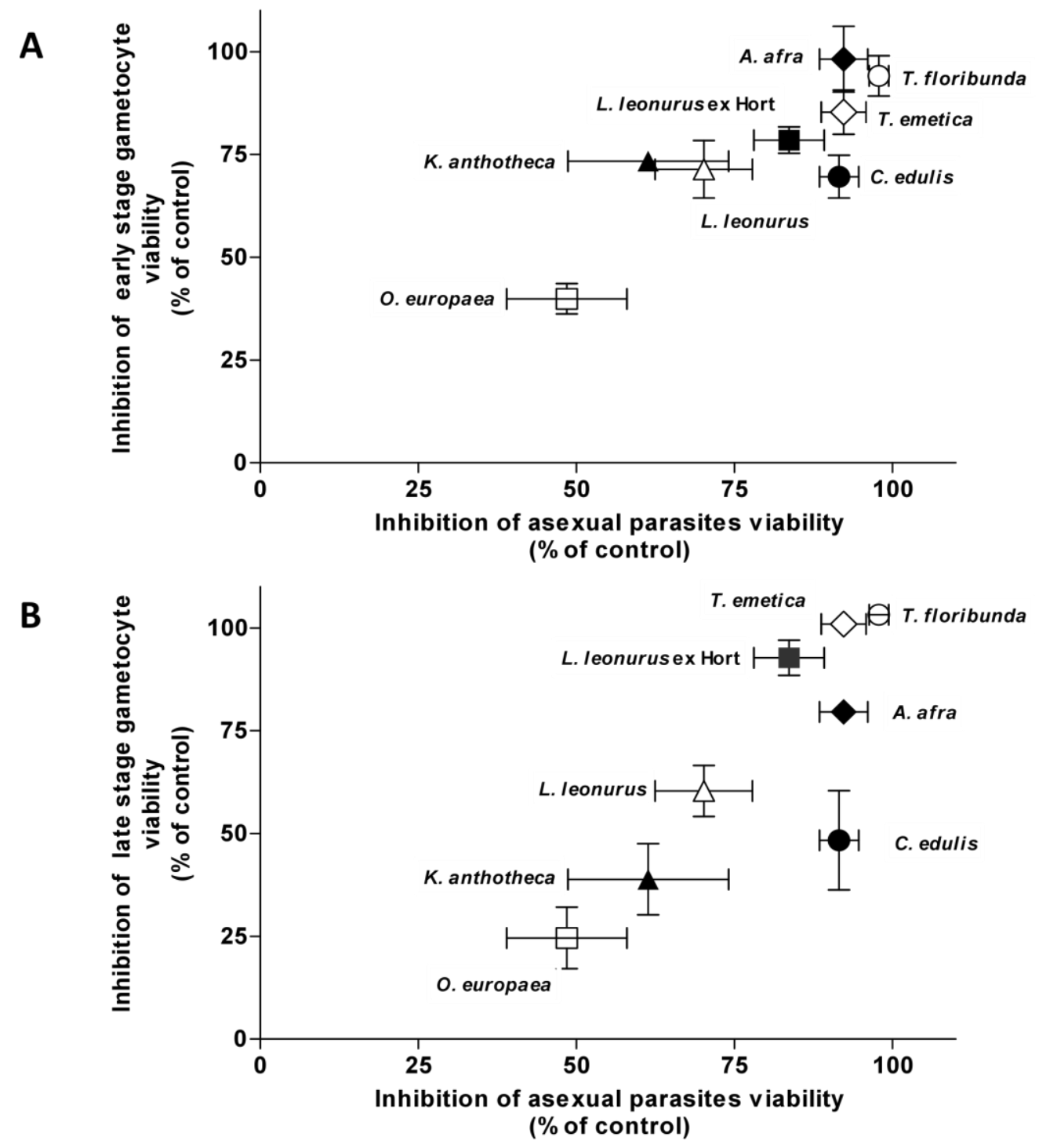

Figure 3. Polypharmacology of crude acetone plant extracts on the in vitro viability of both $\boldsymbol{P}$. falciparum NF54 gametocytes and intraerythrocytic asexual parasites. Plant extracts were screened at $20 \mu \mathrm{g} / \mathrm{ml} \mathrm{using}$ the pLDH assay for both gametocytes and asexual parasites. Inhibition of asexual parasite proliferation was compared to inhibition of either early (A) or late (B) gametocyte viability. Data are expressed as \% viability of untreated controls and are the mean \pm SEM of three independent experiments each done in triplicate. For asexual parasite assays, chloroquine $(0.5 \mu \mathrm{M})$ was used as positive control inhibiting $89.2 \pm 5.3 \%$ parasite viability. 


\section{Discussion}

At the time malaria patients seek medication, there would already be late stage IV and V gametocytes circulating in their system (Eziefula et al., 2014). Late stage gametocytes particularly make a scientifically credible stage to block transmission as they are the only form of $P$. falciparum capable of infecting a female Anopheles mosquito (Sinden et al., 2012a). However, the currently available set of chemotherapeutics is largely only capable of impairing development of early stage gametocytes. The goal of discovering a single drug targeting all malaria stages (Burrows et al., 2013) motivated the evaluation of the ability of plant extracts to inhibit both early and late stage gametocytes in vitro.

There are a number of plants that have been identified and documented in ethnobotanical studies for treating malaria. However, for bio-prospecting purposes, there is need for rational based criteria for prioritising plants to be screened for activity against gametocytes, similar to the approach used for screening against intraerythrocytic asexual parasites (Clarkson et al., 2004). This has the advantage of narrowing down the number of plant species for a study in a systemic manner with a higher probability of getting hits. However, the disadvantage is that other species with gametocytocidal activity may then not be screened. Due to the vast pool of knowledge available on activity of plant extracts against asexual parasites, a criterion was set to screen those with good to moderate activity on asexuals against gametocytes. This approach is also being used in screening currently available malaria treatment drugs against gametocytes (Sinden et al., 2012a). The second selection criterion was based on chemical classes of natural compounds produced by different species of plant families known to have gametocytocidal activity. The importance of extraction method, temperature and solvent is critical with significant consequences on activity as classically illustrated on the role they played in the discovery of artemisinin (Tu, 2011; Zhang, 2011). In our investigation we used a non-destructive shaking method at room temperature (Dzoyem and Eloff, 2015). We selected acetone as the solvent because of its ability to extract many compounds of different polarities from plants, its non-toxic nature and easy removal hence avoiding interference with subsequent assays (Eloff, 1998). 
In this study, extracts from two species of the Meliaceae and one of the Asteraceae plant families had good activity against both early and late stage gametocytes. T. emetica and $T$. floribunda (from the Meliaceae family) both had $\mathrm{IC}_{50}$ values below $10 \mu \mathrm{g} / \mathrm{ml}$ with greater potency towards late stages, which are generally regarded to be the least susceptible to drug treatment (White, 2008). A. indica, a species of the Meliaceae family, has been shown to be equipotent $\left(\mathrm{IC}_{50}=0.001 \mu \mathrm{g} / \mathrm{ml}\right)$ against both early and late gametocyte stages of $P$. falciparum (Udeinya et al., 2006).

The limonoid, azadirachtin, was identified as the active principle in A. indica (Jones et al., 1994). Its mode of action was deciphered to be through an interruption of mitotic spindle formation, a process of great significance in microgametogenesis (Billker et al., 2002). Other limonoids such as gedunin, azadirone and azadiradione from $A$. indica have also been suggested to be gametocytocidal (Yerbanga et al., 2014). The two Meliaceae species used in this study, T. emetica and T. floribunda, are known to produce limonoids, a common feature of this plant family (Roy and Saraf, 2006). This natural product chemical class may be responsible for the gametocytocidal properties of the crude acetone extracts of these plants observed in this study. Both these species were also highly active against intraerythrocytic asexual parasites, a common characteristic of $A$. indica (Udeinya et al., 2006). However, $K$. anthotheca, also a member of the Meliaceae family and a known producer of limonoids (Roy and Saraf, 2006) was shown in this study to have a markedly variable efficacy on multiple stages of the malaria parasite. It has been suggested in other studies that limonoids could act as a scaffold upon which new multiple stage active antimalarial drugs could be designed (Yerbanga et al., 2014). Further enthuse for working on this natural class of compounds is evident from the fact that it also significantly compromises the fitness of the mosquito vector (Dembo et al., 2015).

A. afra (Asteraceae) is a widely used medicinal plant species in South Africa for the treatment of a number of different ailments (Philander, 2011; Van Wyk, 2011, 2008). While it is highly active against the asexual parasites (Clarkson et al., 2004) there has been no report of its potency on P. falciparum NF54 gametocyte stages. However, A. annua (Asteraceae), that produces a sesquiterpene lactone, artemisinin, has gametocytocidal properties in vitro (Duffy and Avery, 2013; Lucantoni et al., 2013). The genus Artemisia is well known for the production of sesquiterpene lactones (Al-khathlan et al., 1992) and A. afra is no exception (Al-khathlan et al., 1992; Jakupovic et al., 1988) but there is no evidence for the presence of artemisinin in the latter specie (Van der Kooy et al., 2008; Liu et al., 2009). To our 
knowledge none of the sesquiterpene lactones (or any other natural compounds from A. afra) were screened for activity against either early or late stage gametocytes of Plasmodium. $V$. amygdalina (Asteraceae) has been shown to be potent against $P$. berghei gametocytes in vivo with two sesquiterpene lactones having been identified as the active compounds (Abay et al., $2015,2013)$. It also has good activity against asexual parasites with an $\mathrm{IC}_{50}$ value of 8.72 $\mu \mathrm{g} / \mathrm{ml}$ against $P$. falciparum 3D7 strain (Zofou et al., 2011). These sesquiterpene lactones may be responsible for the observed inhibition of gametocyte viability by the crude acetone extract of A. afra.

L. leonurus ex Hort (yellow) is a garden cultivar of the wild type L. leonurus (L.) R.Br. There is currently no documented report on the gametocytocidal activity of either of the two species or any family member. In this study, L. leonurus ex Hort (yellow) had the most pronounced activity of the two against both gametocytes and intraerythrocytic asexual parasites. Just as was the case with T. emetica, T. floribunda and A. afra, L. leonurus ex Hort extracts was equally highly potent against both gametocytes and asexual parasites. All plant extracts that were highly active against gametocytes (particularly late stages) were equally active against asexual parasites. This pattern of activity may suggest that selecting plants for screening against gametocytes based on their known activity against asexual parasites may be of merit. However, it must be noted that this observation has been made from a rather small data set and may not necessarily apply to all plant species. It is also worth taking into consideration that A. afra, T. emetica and T. floribunda were also selected based on the classes of compound they are known to produce in addition to inhibition of asexual parasites. Hence, this criterion may also be worth using in selecting other plant species to study.

A drawback of the pLDH assay is its inability to interrogate viability of male and female gametocytes separately in a culture containing both sexes. The implication is that extracts, which may be active against one specific sex, (in which case no complete inhibition is observed) may be flagged as false negatives (Sinden et al., 2012b). Hence extracts from $C$. edulis, L. leonurus and $K$. anthotheca that have been used in this study may still be considered as potential transmission blocking agents. 


\section{Conclusion}

While extensive studies have been carried out in screening extracts of plants against Plasmodium intraerythrocytic asexual parasites, comparatively less screenings have been undertaken against gametocyte stages. The present study has given an insight into the gametocytocidal properties of traditional South African medicinal plant species extracts. To the best of our knowledge, this is the first report on the in vitro inhibition of gametocyte viability by the crude acetone extracts used in this study. Based on the findings of this study and those from the limited literature available, there is merit in channeling more effort towards screening extracts of plants in the transmission blocking agenda. The next step is to isolate and identify the active natural products especially from those plant species, namely $A$. afra, T. emetica and T. floribunda, shown here to inhibit both the asexual and late gametocyte stages. Subsequent studies would include the in vivo "gold standard" membrane-feeding assay, which will indeed provide additional data on the transmission blocking properties of these plant extracts.

\section{Acknowledgements}

We would like to thank Mrs. S. van Wyngaardt for assistance in transportation of plant material and Mr. S. S. Awandu and Dr. A. Andayi for helping collect plant material. The authors are also grateful to Mr. J. Sampson (Curator at Manie van der Schijff Botanical Garden at the University of Pretoria) for his assistance in plant identification and collection. We would further like to acknowledge staff at the H.G.W.J. Schweickerdt Herbarium of the University of Pretoria for receiving and preparing our voucher specimens. This work was supported by a research grant from the University of Pretoria Centre for Sustainable Malaria Control (UP CSMC), the South African National Research Foundation (UID:84627), and the Medical Research Council Strategic Health Innovation Partnership. 


\section{7. $\quad$ References}

Abay, S.M., 2013. Blocking malaria transmission to Anopheles mosquitoes using artemisinin derivatives and primaquine: a systematic review and meta-analysis. Parasite and Vectors $6,278$.

Abay, S.M., Dahiya, N., Dori, G., Dembo, E., Ogboi S., Lupidi, G. et al., 2013. Malaria transmission blocking potential of Vernonia amygdalina (Grawa) leaves. Poster presentation at 6th Pan-African MIM Conference, Durban; and Gordon Malaria conference 2013.

Abay, S.M., Lucantoni, L., Dahiya, N., Dori, G., Dembo, E.G., Esposito, F. et al., 2015. Plasmodium transmission blocking activities of Vernonia amygdalina extracts and isolated compounds. Malaria Journal 14, 288.

Adjalley, S.H., Johnston, G.L., Li, T., Eastman, R.T., Ekland, E.H., Eappen, A.G. et al., 2011. Quantitative assessment of Plasmodium falciparum sexual development reveals potent transmission-blocking activity by methylene blue. Proceedings of the National Academy of Sciences 108, E1214-E1223.

Alam, M.K., Basak, S.R., Alam, S., 2012. Khaya anthotheca (Welw.) C. DC. (Meliaceae)-An exotic species in Bangladesh. Bangladesh Journal of Plant Taxonomy 19, 95-97.

Al-Khathlan, H.Z., Mousa, A.A., Al-Hazimi, H.M.G., 1992. Sesquiterpene lactones from Artemisia species. Journal of the Chemical Society of Pakistan 14, 151-165.

Alonso, P.L., Brown, G., Arevalo-Herrera, M., Binka, F., Chitnis, C., Collins, F. et al., 2011. A research agenda to underpin malaria eradication. PLoS Medicine, 8.

Ashley, E.A., Dhorda, M., Fairhurst, R.M., Amaratunga, C., Lim, P., Suon, S. et al., 2014. Spread of artemisinin resistance in Plasmodium falciparum malaria. New England Journal of Medicine 371, 411-423.

Baird, J.K., Hoffman, S.L., 2004. Primaquine Therapy for Malaria. Clinical Infectious Diseases 39, 1336-1345.

Baker, D.A., 2010. Malaria gametocytogenesis. Molecular and biochemical Parasitology 172, 57-65.

Benelli, G., 2015. Research in mosquito control: current challenges for a brighter future. Parasitology Research 114, 2801-2805.

Bhatt, S., Weiss, D.J., Cameron, E., Bisanzio, D., Mappin, B., Dalrymple, U. et al., 2015. The effect of malaria control on Plasmodium falciparum in Africa between 2000 and 2015. Nature 526, 207-211.

Billker, O., Shaw, M.K., Jones, I.W., Ley, S.V., Mordue, A.J, Sinden, R.E., 2002. Azadirachtin disrupts formation of organised microtubule arrays during microgametogenesis of Plasmodium berghei. Journal of Eukaryotic Microbiology 49, 489-497.

Burrows, J.N., van Huijsduijnen, R.H., Möhrle, J.J., Oeuvray, C., Wells, T.N., 2013. Designing the next generation of medicines for malaria control and eradication. Malaria Journal 12, 10-1186.

Butcher, G.A., 1997. Antimalarial drugs and the mosquito transmission of Plasmodium. International Journal for Parasitology 27, 975-987.

Clarkson, C., Campbell, W.E., Smith, P., 2003. In vitro antiplasmodial activity of abietane and totarane diterpenes isolated from Harpagophytum procumbens (Devil's Claw). Planta Medica 8, 720-724.

Clarkson, C., Maharaj, V.J., Crouch, N.R., Grace, O.M., Pillay, P., Matsabisa, M.G. et al., 2004. In vitro antiplasmodial activity of medicinal plants native to or naturalised in South Africa. Journal of Ethnopharmacology 92, 177-191. 
Dai, Y., Huang, X., Cheng, P., Liu, L., Wang, H., Wang, H., Kou, J., 2015. Development of insecticide resistance in malaria vector Anopheles sinensis populations from Shandong province in China. Malaria Journal 14, 62.

D'Alessandro, S., Corbett, Y., Ilboudo, D.P., Misiano, P., Dahiya, N., Abay, S.M. et al., 2015. Salinomycin and other ionophores as a new class of antimalarial drugs with transmission-blocking activity. Antimicrobial Agents and Chemotherapy 59, 5135-5144.

D'Alessandro, S., Silvestrini, F., Dechering, K., Corbett, Y., Parapini, S., Timmerman, M. et al., 2013. A Plasmodium falciparum screening assay for anti-gametocyte drugs based on parasite lactate dehydrogenase detection. Journal of Antimicrobial Chemotherapy 68, 2048-2058.

Dembo, E.G., Abay, S.M., Dahiya, N., Ogboi, J.S., Christophides, G.K., Lupidi, G. et al., 2015. Impact of repeated NeemAzal ${ }^{\circledR}$-treated blood meals on the fitness of Anopheles stephensi mosquitoes. Parasites and Vectors 8, 94.

Dhar, R., Zhang, K., Talwar, G.P., Garg, S., Kumar, N., 1998. Inhibition of the growth and development of asexual and sexual stages of drug-sensitive and resistant strains of the human malaria parasite Plasmodium falciparum by Neem (Azadirachta indica) fractions. Journal of Ethnopharmacology 61, 31-39.

Dondorp, A.M., Nosten, F., Yi, P., Das, D., Phyo, A. P., Tarning, J. et al., 2009. Artemisinin resistance in Plasmodium falciparum malaria. New England Journal of Medicine 361, 455-467.

Duffy, S., Avery, V., 2013. Identification of inhibitors of Plasmodium falciparum gametocyte development. Malaria Journal 12, 408.

Dzoyem, J.P., Eloff, J.N., 2015. Anti-inflammatory, anticholinesterase and antioxidant activity of leaf extracts of twelve plants used traditionally to alleviate pain and inflammation in South Africa. Journal of Ethnopharmacology 160, 194-201.

Edi, C.V., Koudou, B.G., Jones, C.M., Weetman, D., Ranson, H., 2012. Multiple-insecticide resistance in Anopheles gambiae mosquitoes, Southern Cote d'Ivoire. Emerging Infectious Diseases 18, 1508.

Eloff, J.N., 1998. Which extractant should be used for the screening and isolation of antimicrobial components from plants? Journal of Ethnopharmacology 60, 1-8.

Eziefula, A.C., Bousema, T., Yeung, S., Kamya, M., Owaraganise, A., Gabagaya, G. et al., 2014. Single dose primaquine for clearance of Plasmodium falciparum gametocytes in children with uncomplicated malaria in Uganda: a randomised, controlled, double-blind, dose-ranging trial. The Lancet Infectious Diseases 14, 130-139.

Jakupovic, J., Klemeyer, H., Bohlmann, F., Graven, E.H., 1988. Glaucolides and guaianolides from Artemisia afra. Phytochemistry 4, 1129-1133.

Jones, I.W., Denholm, A.A., Ley, S.V., Lovell, H., Wood, A., Sinden, R.E., 1994. Sexual development of malaria parasites is inhibited in vitro by the neem extract azadirachtin, and its semi-synthetic analogues. FEMS Microbiology Letters 120, 267-273.

Kotze, M., Eloff, J.N., 2002. Extraction of antibacterial compounds from Combretum microphyllum (Combretaceae). South African Journal of Botany 68, 62-67.

Liu, N.Q., Van der Kooy, F., Verpoorte, R., 2009. Artemisia afra: a potential flagship for African medicinal plants? South African Journal of Botany 75, 185-195.

Lucantoni, L., Duffy, S., Adjalley, S.H., Fidock, D.A., Avery, V.M., 2013. Identification of MMV malaria box inhibitors of Plasmodium falciparum early-stage gametocytes using a luciferase-based high-throughput assay. Antimicrobial Agents and Chemotherapy 57, 6050-6062.

Lucantoni, L., Yerbanga, R.S., Lupidi, G., Pasqualini, L., Esposito, F., Habluetzel, A., 2010. Transmission blocking activity of a standardized neem (Azadirachta indica) seed extract 
on the rodent malaria parasite Plasmodium berghei in its vector Anopheles stephensi. Malaria Journal 9, 66.

Mackerras, M.J., Ercole, Q.N., 1949. Observations on the action of quinine, atebrin and plasmoquine on the gametocytes of Plasmodium falciparum. Transactions of the Royal Society of Tropical Medicine and Hygiene 42, 455-463.

Makler, M.T., Hinrichs, D.J., 1993. Measurement of the lactate dehydrogenase activity of Plasmodium falciparum as an assessment of parasitemia. American Journal of Tropical Medicine and Hygiene 48, 205-210

Nkhoma, E.T., Poole, C., Vannappagari, V., Hall, S.A., Beutler, E., 2009. The global prevalence of glucose-6-phosphate dehydrogenase deficiency: a systematic review and meta-analysis. Blood Cells, Molecules and Diseases 42, 267-278.

Philander, L.A., 2011. An ethnobotany of Western Cape Rasta bush medicine. Journal of Ethnopharmacology 138, 578-594.

Price, R.N., Nosten, F., Luxemburger, C., Ter Kuile, F.O., Paiphun, L., Chongsuphajaisiddhi, T., White, N.J., 1996. Effects of artemisinin derivatives on malaria transmissibility. The Lancet 347, 1654-1658.

Ranson, H., N'Guessan, R., Lines, J., Moiroux, N., Nkuni, Z., Corbel, V., 2011. Pyrethroid resistance in African anopheline mosquitoes: what are the implications for malaria control? Trends in Parasitology 27, 91-98.

Reader, J., Botha, M., Theron, A., Lauterbach, S.B., Rossouw, C., Engelbrecht, D. et al., 2015. Nowhere to hide: interrogating different metabolic parameters of Plasmodium falciparum gametocytes in a transmission blocking drug discovery pipeline towards malaria elimination. Malaria Journal 14, 213.

Roberts, L., Enserink, M., 2007. Did they really say. . .eradication? Science 318, 1544-1545.

Roy, A., Saraf, S., 2006. Limonoids: overview of significant bioactive triterpenes distributed in plants kingdom. Biological and Pharmaceutical Bulletin 29, 191-201.

Sáenz, F.E., LaCrue, A.N., Cross, R.M., Maignan, J.R., Udenze, K.O., Manetsch, R. et al., 2013. 4- $(1 \mathrm{H})$-quinolones and 1, 2, 3, 4-tetrahydroacridin-9 (10H)-ones prevent the transmission of Plasmodium falciparum to anopheles freeborni. Antimicrobial Agents and Chemotherapy 57, 6187-6195.

Sinden, R.E., Blagborough, A.M., Churcher, T., Ramakrishnan, C., Biswas, S., Delves, M.J., $2012 \mathrm{~b}$. The design and interpretation of laboratory assays measuring mosquito transmission of Plasmodium. Trends in Parasitology 28, 457-465.

Sinden, R.E., Carter, R., Drakeley, C., Leroy, D., 2012a. The biology of sexual development of Plasmodium: the design and implementation of transmission-blocking strategies. Malaria Journal 11, 1-11.

Sun, W., Tanaka, T.Q., Magle, C.T., Huang, W., Southall, N., Huang, R., 2014. Chemical signatures and new drug targets for gametocytocidal drug development. Scientific Reports 4.

Tu, Y., 2011. The discovery of artemisinin (qinghaosu) and gift from Chinese medicine. Nature Medicine 17, 19-22.

Tun, K.M., Imwong, M., Lwin, K.M., Win, A.A., Hlaing, T.M., Hlaing, T. et al., 2015. Spread of artemisinin-resistant Plasmodium falciparum in Myanmar: a cross-sectional survey of the K13 molecular marker. The Lancet Infectious Diseases 15, 415-421.

Udeinya, I.J., Brown, N, Shu, E.N., Udeinya, F.I., Quakyi, I., 2006. Fractions of an antimalarial neem leaf extract have activities superior to chloroquine, and are gametocytocidal. Annals of Tropical Medicine and Parasitology 100, 17-22.

Udeinya, I.J., Shu, E.N., Quakyi, I., Ajayi, F.O., 2008. An antimalarial neem leaf extract has both schizonticidal and gametocytocidal activities. American Journal of Therapeutics 15, 108-110. 
Van der Kooy, F., Verpoorte, R., Meyer, J.M., 2008. Metabolomic quality control of claimed anti-malarial Artemisia afra herbal remedy and A. afra and A. annua plant extracts. South African Journal of Botany 74, 186-189.

Van Wyk, B.E., 2008. A broad review of commercially important southern African medicinal plants. Journal of Ethnopharmacology 119, 342-355.

Van Wyk, B.E., 2011. The potential of South African plants in the development of new medicinal products. South African Journal of Botany 77, 812-829

Verlinden, B.K., Niemand, J., Snyman, J., Sharma, S.K., Beattie, R.J., Woster, P.M., Birkholtz, L.M., 2011. Discovery of novel alkylated (bis) urea and (bis) thiourea polyamine analogues with potent antimalarial activities. Journal of Medicinal Chemistry 54, 6624-6633.

Wells, T.N., 2011. Natural products as starting points for future anti-malarial therapies: going back to our roots. Malaria Journal 10, S3.

White, N.J., 2008. The role of anti-malarial drugs in eliminating malaria. Malaria Journal 7 , S8.

White, N.J., Ashley, E.A., Recht J., Delves, M.J., Ruecker, A., Smithuis, F.M. et al., 2014. Assessment of therapeutic responses to gametocytocidal drugs in Plasmodium falciparum malaria. Malaria Journal 13, 483.

Yerbanga, R.S., Lucantoni, L., Ouédraogo, R.K., Da, D.F., Yao, F.A., Yaméogo, K.B. et al., 2014. Transmission blocking activity of Azadirachta indica and Guiera senegalensis extracts on the sporogonic development of Plasmodium falciparum field isolates in Anopheles coluzzii mosquitoes. Parasites and Vectors 7, 185.

Zhang, X., 2011. Youyou Tu honored by the Lasker award. Protein Cell 2, 773.

Zofou, D., Tene, M., Ngemenya, M.N., Tane, P., Titanji, V.P., 2011. In vitro antiplasmodial activity and cytotoxicity of extracts of selected medicinal plants used by traditional healers of Western Cameroon. Malaria Research and Treatment, Article ID 561342. 


\section{Supplementary material}

A

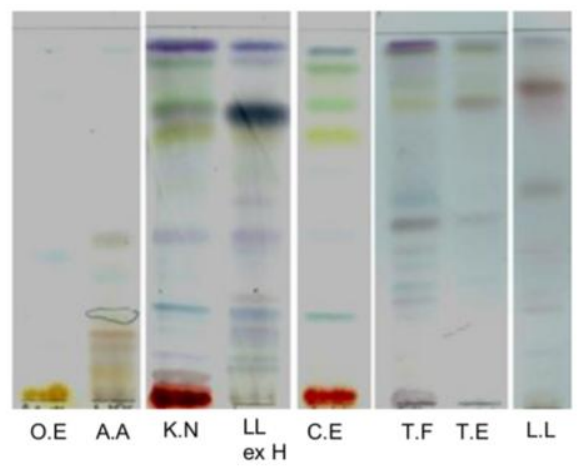

B

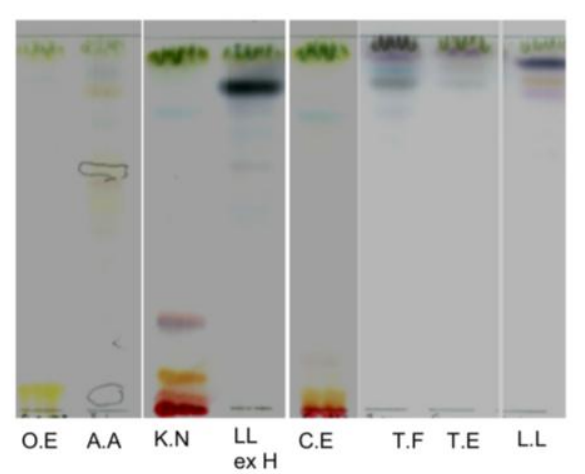

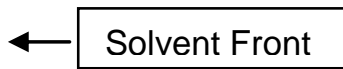

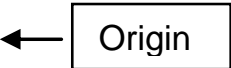

C

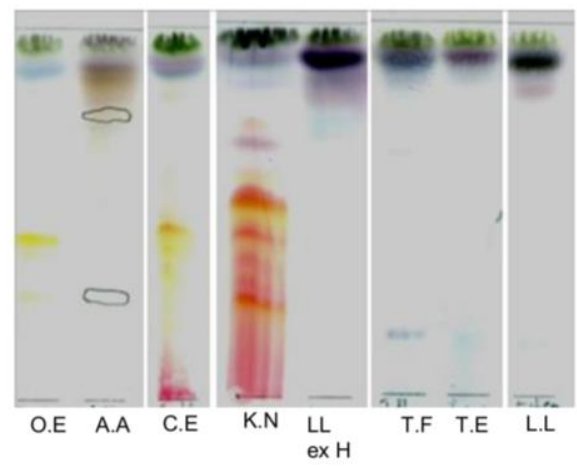

Figure 1S. Thin-layer chromatograms of crude acetone extracts from plants. Three different mobile solvent systems were used for developing the chromatograms. (A) benzene, ethanol and ammonium hydroxide solution (90:10:1), (B) chloroform, ethyl acetate and formic acid solution (50:40:10) and (C) ethyl acetate, methanol and water solution (40:6.5:5). Compounds were visualised with vanillin-sulphuric acid (Kotze and Eloff, 2002). Major compound classes identified include include phenols (yellow), terpenoids (purple or bluish purple) and stilbenes (bright red). A. afra was the only species observed to have compounds that fluoresce under UV light (circled) (O.E - Olea europaea; A.A - Artemisia afra; K.N - Khaya anthotheca; L.L ex H - Leonotis leonurus ex Hort; C.E - Catha edulis; T.F - Turraea floribunda; T.E - Trichilia emetica; L.L - Leonotis leonurus) 